\title{
Effect of processing conditions on the mechanical behavior of amino functionalized nanotube modified glass fiber epoxy composites
}

\begin{abstract}
In the present study, 2-ply glass fiber epoxy composites were prepared with aminofunctionalized carbon nanotubes using Vacuum assisted resin infusion molding (VARIM) technique. The focus is to evaluate the different processing techniques used to uniformly disperse nanotubes in resin and their effect on mechanical properties. In addition, the dispersion and mechanical performance by incorporating aminonanotubes in curing agent vis-à-vis epoxy resin is also studied. Lastly, the mechanical behavior of composites on increasing the resin infusion temperature prior to its fabrication is also investigated. The mechanical results along with SEM and TEM images validate the proposed processing methodology and use of higher infusion temperature.
\end{abstract}

Keywords: carbon nanotubes, epoxy, glass fiber, mechanical properties, VARIM
Volume I Issue 2 - 2017

\author{
Mohit Garg, Shruti Sharma, ${ }^{2}$ Rajeev Mehta ${ }^{3}$ \\ 'Department of Civil Engineering, University of Victoria,Victoria, \\ Canada \\ ${ }^{2}$ Department of Civil Engineering, Thapar University, India \\ ${ }^{3}$ Department of Chemical Engineering, Thapar University, USA
}

Correspondence: Mohit Garg, Department of Civil

Engineering, University of Victoria, Victoria, British Columbia,

Canada,Email mgarg@uvic.ca

Received: June 13,2017| Published: July 20, 2017

\section{Introduction}

The polymer nanocomposites (PNC) prepared using nanofillers such as carbon nanotubes and nanoclay has shown significant improvement in the mechanical properties. ${ }^{1-5}$ Carbon nanotube reinforced glass fiber polymer composites exhibit high strength to weight ratio, superior corrosion and fatigue resistance as compared to neat epoxy composites likely due to their intrinsic properties.

In the last decade, CNTs have shown wide applications in the areas of aerospace, automotive and marine structures as the CNT nanocomposites properties scored over other nano-fillers with respect to mechanical, electrical and thermal properties. The superior mechanical properties of CNT have allowed them to be used as secondary reinforcement in composite materials. Further improvement in mechanical properties has been reported after the addition of amino functionalized CNT in PNC. . $^{1,-5,6-20}$ Besides, effective interfacial bonding between the composites individual constituents the properties of the composite materials is primarily dependent on the dispersion of CNT in the polymer matrix. Thus, at present the full potential of CNTs as reinforcement is still limited due to the non-homogenous dispersion of CNT as they exist as agglomerates and also due to poor interfacial interaction between CNTs and the polymer matrix. ${ }^{21}$ There is an enormous amount of literature available on the techniques developed for CNT dispersion in polymer matrix. ${ }^{22-27}$ From the literature it has been deduced that 'ultrasonication' is an effective method to disperse CNTs in liquids having a low viscosity, such as water, solvents and few polymer matrices. In addition to that, mechanical stirring has also been considered as an effective tool to disperse nanoparticles in a polymer matrix. The size and shape of the propeller and the mixing speed controls the dispersion results. A study May $\mathrm{CA}^{28}$ has reported a relatively fine dispersion of CNT in polymer matrix after intensive stirring. On the other hand, using a homogenizer to induce high shear rates that assist in obtaining uniform particle size and dispersion of nanoparticles has not been studied much. The literature supporting the use of homogenizer is very scant and thus it is interesting to find out if mechanical stirring or high-shear homogenization would result in uniform dispersion of CNTs in the epoxy resin and a stabilized mixture i.e. without re-agglomeration of CNTs when kept for longer durations.

In the present study, the effect of various parameters on the mechanical properties of CNT modified FRP composites have been studied. This includes studying the effect of mechanical stirring as well as high-shear homogenization to uniformly disperse aminofunctionalized nanotubes (ACNT) in the resin on the tensile and flexural properties. Secondly, the degree of dispersion and interfacial bonding of ACNT in epoxy resin or amine hardener has also been looked into. Lastly, the effect of temperature of final polymer mixture during infusion in VARIM has also been considered. The investigations of all these parameters lead to optimization of the conditions that significantly affect the dispersion, interfacial bonding and the resulting mechanical properties of the polymer nanocomposites. Furthermore, electron microscopy techniques validates the dispersion of carbon nanotubes in polymer matrix and the presence of strong bonding that possibly assisted in improving the strength and stiffness of the prepared composite material.

\section{Experimental}

\section{Materials}

The details of procurement of raw materials for the preparation of polymer nanocomposites have been documented in one of our earlier work. $^{3}$

\section{Preparation of glass fiber polymer composites}

The preparation of pure epoxy and CNT reinforced epoxy GFRP composites have been reported in detail in Garg $\mathrm{M}^{3}$ and a schematic diagram for the same is shown in Figure 1.

In brief, the processing methodology to obtain uniform and homogenous dispersion of nanotubes in epoxy matrix has two methods, firstly, homogenization and ultrasonication (Figure 1) and secondly, stirring and ultrasonication. The polymer nanocomposites 
(PNC's) were prepared by adding 0.1 wt $\%$ amino-functionalized CNT (ACNT) in epoxy resin using either homogenizer at a speed of 20,000 rpm or mechanical stirrer at $500 \mathrm{rpm}$. The resulting CNT/ epoxy mixture was sonicated at $40 \%$ amplitude for $10 \mathrm{~min}$ followed by the addition of curing agent in stoichiometric ratio, using mechanical stirrer. Lastly, the polymer mixture was infused into the mold using VARIM for fabricating CNT reinforced glass fiber composite material.

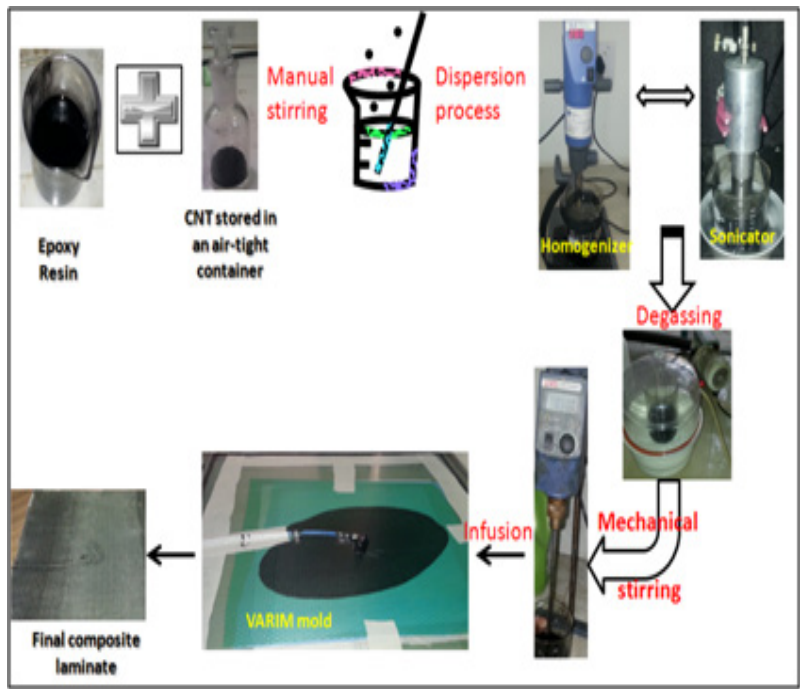

Figure I Schematic of the process used to prepare polymer nanocomposite.

The morphological characterization of the fractured surfaces of the samples was done in scanning electron microscopy - SEM (JEOL JSM 6510LV SEM at 20kV). All the specimens were prepared with a thin gold coating to avoid charging effects. The dispersion was quantified by analyzing the liquid samples under transmission electron microscope, Hitachi (H-7500), after placing onto a copper grid. The tests for both mechanical properties (i.e. tensile and flexural) were carried out on a universal testing machine, Zwick Roell Z010. The tensile tests were carried according to ASTM D3039 at a crosshead speed (strain rate) of $2 \mathrm{~mm} / \mathrm{min}$ while the flexural tests were performed at a strain rate of $1.34 \mathrm{~mm} / \mathrm{min}$ (ASTM D790). For each prepared composition, four samples were tested.

\section{Results and discussion}

\section{Effect of processing techniques on CNT dispersion in epoxy matrix}

The processing methodology adopted to obtain uniform and homogenous dispersion of nanotubes in epoxy matrix has been defined in two ways: (i) homogenization and ultrasonication (Figure 1), (ii) stirring and ultrasonication. The experimental results obtained after performing mechanical tests of the prepared samples have been illustrated in the Figure 2 and Figure 3.

It is clearly seen that that flexural modulus and strength decreased by $25 \%$ and $29 \%$ respectively, for the mechanical stirring as compared to high-shear homogenization. Homogenization has scored over conventional mechanical stirring technique due to the fact that highshear forces are needed to achieve a fine dispersion in the polymer matrix especially for these thermosetting polymers. Use of a high shear homogenizer at a speed of 20,000 rpm resulted in breaking the nanotube agglomerates and further improved the interfacial bonding with the epoxy resin that enabled an effective stress transfer between the epoxy matrix and the amino-functionalized CNTs. This is clearly represented by the significant enhancement in the mechanical properties, especially flexural ones, obtained by the use of high-shear homogenization as shown in Figure 2 and Figure 3 and the TEM image (Figure 4). The changes observed in flexural properties illustrate the presence of strong interfacial bonding between the CNT reinforced resin and the glass fiber mat.

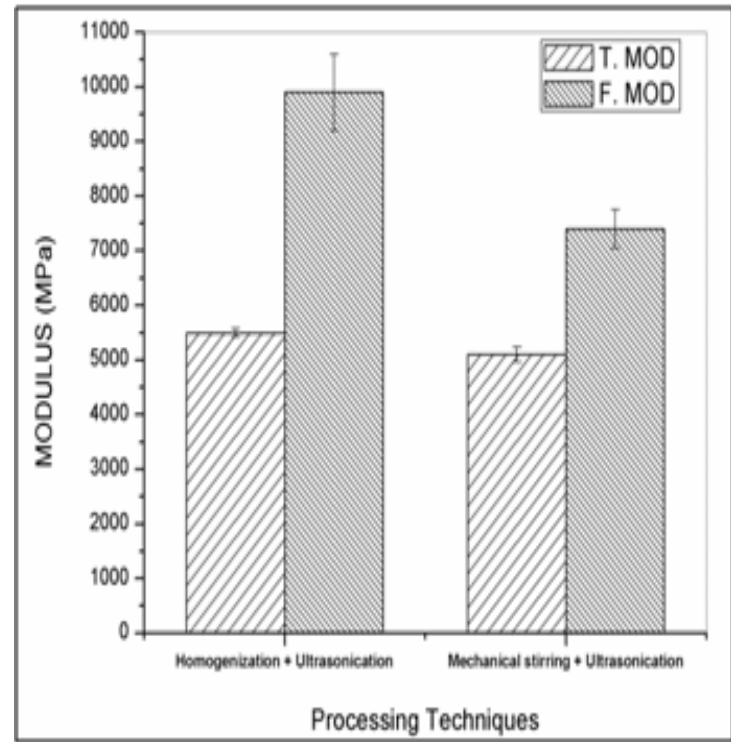

Figure 2 Effect of processing techniques on the modulus of CNT reinforced composites.

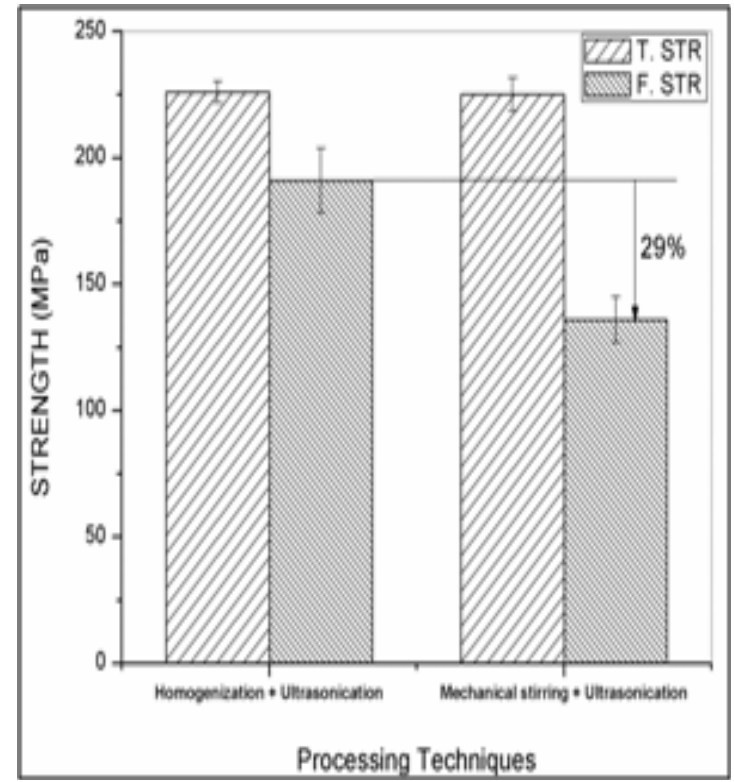

Figure 3 Effect of processing techniques on the strength of CNT reinforced composites.

Figure 4 illustrates the uniformly dispersed ACNT throughout the epoxy resin without altering their original aspect ratio. On comparing the properties obtained by implementing high-shear homogenization with the pure epoxy glass fiber composite (GFRP) an improvement of $36 \%, 45 \%$ and $39 \%$ in flexural modulus, strength and ILSS has been observed, respectively. Whereas, the improvement is only $3 \%$ in the flexural strength for samples prepared with mechanical stirring vis-à- 
vis GFRP composite. This is possibly due to the fact that stirring may not be able to break the CNT agglomerates which would act as stressconcentration sites in the composite. During the testing conditions, these sites become source of crack initiation and finally failure. Hence, these experimental findings suggest the use of homogenization over mechanical stirring for dispersing CNTs in the epoxy resin.

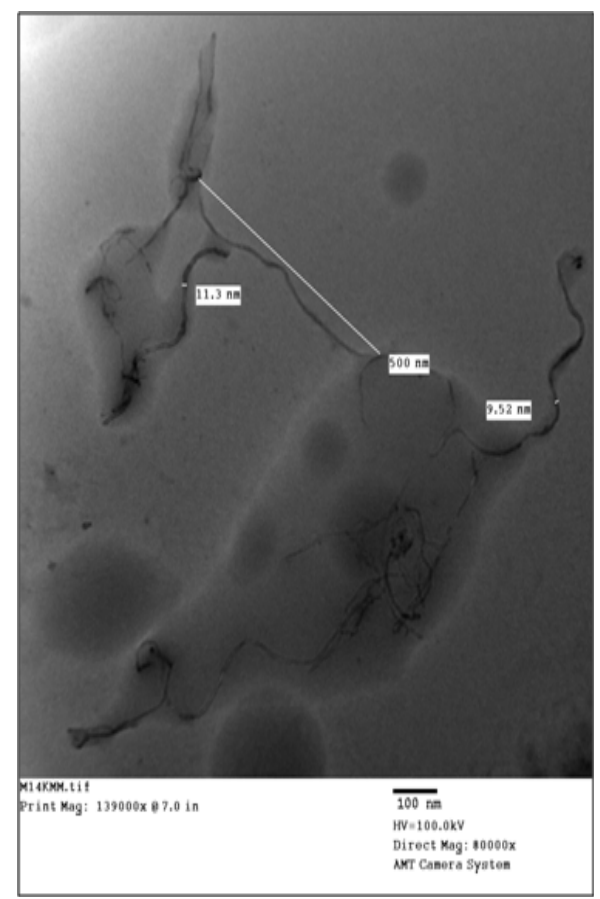

Figure 4 Dispersion of amino-CNTs in epoxy resin characterized using TEM.

\section{Effect of ACNT dispersion in amine hardener versus resin}

The hardener used in the study has diamine groups and is of low viscosity. Instead of dispersing CNT in epoxy resin, researchers have also dispersed them in hardener using processing techniques such as sonication, shear-mixing or calendaring. ${ }^{13,29}$ In the present study, $0.1 \mathrm{wt} \%$ ACNT of the total mixture was added to the hardener using high-shear homogenizer at $10,000 \mathrm{rpm}$ for $5 \mathrm{~min}$ followed by probe sonication set at $40 \%$ amplitude for $10 \mathrm{~min}$. The resulting mixture was kept in a desiccator under vacuum for $1 \mathrm{~h}$ to remove any entrapped air. The stoichiometric ratio of resin was then added using a mechanical stirrer at 500rpm for $5 \mathrm{~min}$. The resulting mixture was cooled down to approximately $30^{\circ} \mathrm{C}$ to prepare FRP reinforced composite material Four samples were tested (as per ASTM standards) under tensile and flexural loading conditions. According to the experimental results obtained (Table 1), there is a remarkable improvement in the mechanical properties as compared to the sample prepared by dispersing ACNT in epoxy resin. Also, the tensile and flexural modulus and strength are higher as compared to neat epoxy composites.

Table 1 reveals significant improvement in the tensile properties by the addition of nanotubes in hardener, as the tensile properties are matrix dominated. The matrix showed better dispersion and strong interfacial attraction between the ACNT particles and the matrix as seen in Figure 4. The fiber-dominated properties i.e. flexural, on the other hand showed little improvement in the flexural strength and ILSS. These results (Table 1) suggest that the addition of ACNT in hardener has shown significant improvement as compared to the nanocomposites prepared by dispersing ACNT in resin and even considerable increase in flexural properties on comparing with neat epoxy composite. The possible explanation for such performance is that the addition of amino-functionalized CNTs in a hardener, low viscosity, containing similar amino groups builds strong covalent bonds among themselves due to the same polarity and formed a long chain. This polymer chain then had abundant amino groups present in the mixture and acted as a nucleophile when exposed to the epoxide groups present in the mixture thus, resulting in the formation of strong interconnected network. Additionally, there would be covalent bonds between the amino CNTs and the resin which further improved the fiber-matrix interfacial bonding. The presence of strong interfacial bonding between the ACNT and epoxy has proven to be beneficial for effective stress transfer under the loading conditions and thus resulted in an enhancement in tensile and flexural properties. This is certainly an interesting result which can further be correlated by examining the fracture surface analysis using SEM and morphological analysis by TEM.

Table I Comparison of the mechanical properties by dispersing ACNT in hardener

\begin{tabular}{lll}
\hline Properties & $\begin{array}{l}\text { \% Change as } \\
\text { compared to } \\
\text { ACNT dispersion } \\
\text { in resin }\end{array}$ & $\begin{array}{l}\text { \% Improvement as } \\
\text { compared to neat epoxy } \\
\text { without nanofillers }\end{array}$ \\
\hline $\begin{array}{l}\text { Tensile modulus } \\
\text { Flexural } \\
\text { modulus }\end{array}$ & $30 \%$ increase & $11 \%$ increase \\
$\begin{array}{l}\text { Tensile strength } \\
\text { Flexural }\end{array}$ & $42 \%$ increase & $20 \%$ increase \\
strength & $6.1 \%$ increase & $54 \%$ increase \\
ILSS & $12 \%$ increase & $55 \%$ increase \\
\hline
\end{tabular}

From Figure 5a, it is clearly visible that the bi-directional layers of the glass fiber mat have a coarse surface. The surface appears to be denser and compact with unbroken CNTs still adhering to the matrix thus suggesting comparatively stronger interfacial bonding between the resin and the amino-functionalized CNTs. On the other hand, Figure 5B shows even denser surface. The fibers are covered by the matrix completely. Moreover, it can be seen that even after the application of compressive stress, the fibers direction has not changed. Generally, it is observed that on application of stress on the composites, the fibers tend to break and appear as broken pieces as seen in Figure 5A. This has not been observed in this case which is certainly due to the existence of strong bonding between the matrix, glass fibers and the nanotubes. The strong interface resulted in an effective stress-transfer during the mechanical testing. The strength of these composites is therefore comparatively higher possibly due to the strong interaction between functionalized nanotubes and the amine hardener. Similar fracture surface behavior by suspending $\mathrm{ACNT}$ in the hardener has been reported by Gojny FH. ${ }^{13}$

\section{Effect of resin mixture temperature during infusion on mechanical properties}

Another important variable which possibly affects the performance of the polymer based composites is their infusion temperature. The temperature of the polymer matrix prior to infusion affects its 
viscosity which indirectly affects the materials mechanical properties. Hence, to investigate this, experiments were carried out by increasing the temperature to $45^{\circ} \mathrm{C}$ from the room temperature (i.e. $23^{\circ} \mathrm{C}-26^{\circ} \mathrm{C}$ ). To validate this further, a comparison was made for two different polymer compositions i.e. $0.0 \% \mathrm{CNT}$ (pure epoxy) and $0.1 \mathrm{wt} \% \mathrm{ACNT}$ epoxy composites. The stiffness and strength obtained for the tested samples are shown in Figure 6 \& Figure 7. In the Figure 6 and Figure 7 GFRP refer to the pure epoxy glass fiber composites without any nanofiller content while, ACNT GFRP refers to glass fiber composites prepared where amino-functionalized nanotubes act as secondary reinforcement material.

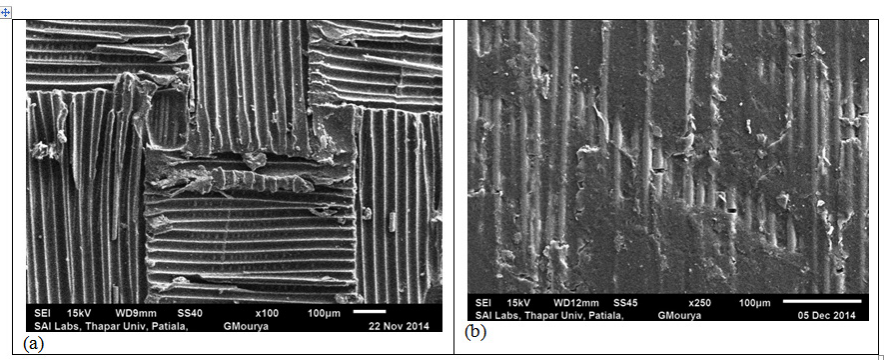

Figure 5 SEM images of (a) ACNT dispersion in resin and (b) ACNT dispersion in hardener.

It is clearly visible that as the infusion temperature is increased above the room temperature up to $45^{\circ} \mathrm{C}$, it has a strong positive effect on the stiffness especially for pure epoxy (GFRP) composite samples while, for ACNT GFRP composites there was a fall in modulus values as seen in Figure 6. The mechanical behavior of composites during tensile and compressive loading conditions reveals a slight enhancement in the strength properties vis-à-vis reference specimen as seen in Figure 7. This is certainly an interesting finding because at room temperature $\mathrm{CNT} /$ epoxy mixture is highly viscous which results in increasing the possibility of formation of CNT agglomerates during its curing. In addition, Figure 8 reveals an inverse relation between strain and modulus for ACNT GFRP composition i.e. strain increases while modulus decreases for samples prepared at $45^{\circ} \mathrm{C}$ vis-à-vis $23^{\circ} \mathrm{C}$ samples. Similar behavior has also been observed for other properties i.e. tensile strength, flexural strength and ILSS properties. In general, the higher infusion temperature of mixture has proven to be advantageous for GFRP specimens with respect to all mechanical properties, while it is specific to few properties in the case of ACNT GFRP specimens. Therefore, the fabrication of polymer nanocomposites should certainly consider higher temperature of resin mixture prior to its infusion for curing as per the findings from this experimental study.

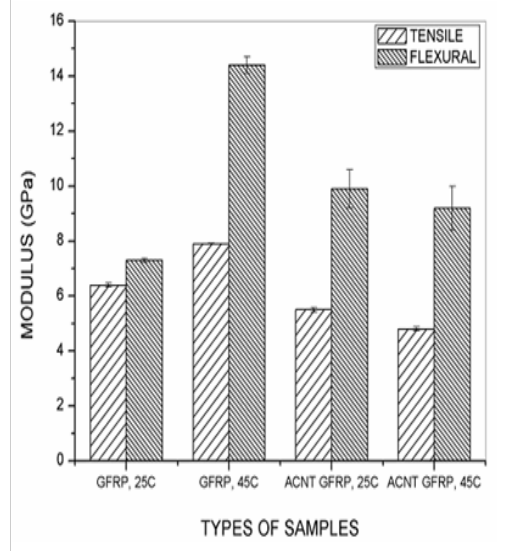

Figure 6 Effect of polymer mixture temperature on composites modulus.
But on the other hand, infusion at room temperature results in higher viscosity of the CNT/epoxy mixture that cause poor wetting of the glass fiber mat, as it becomes more difficult for the partially crosslinked polymer molecules to conform to the fiber mat and infiltrate the inter-strand and inter-filament spaces. ${ }^{29}$ This leads to increased porosity and defect sites in the resulting composites that eventually become the source of crack initiation in the composite under stress and the ultimate reason for the failure of the composites.

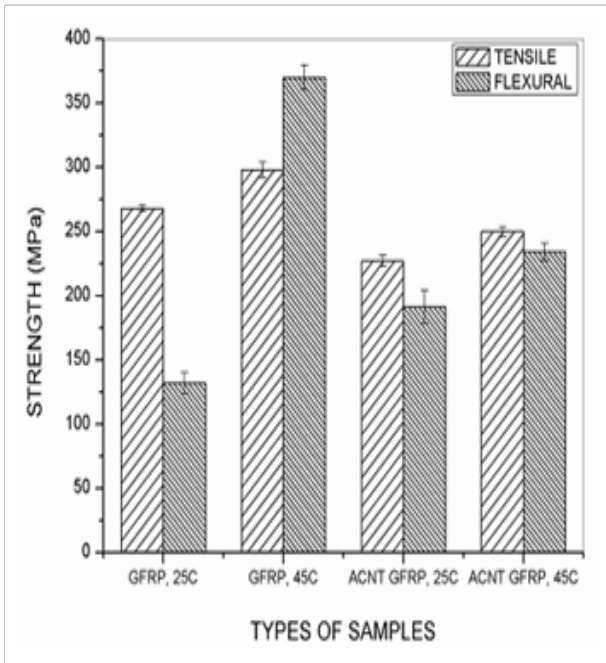

Figure 7 Effect of polymer mixture temperature on composites strength.

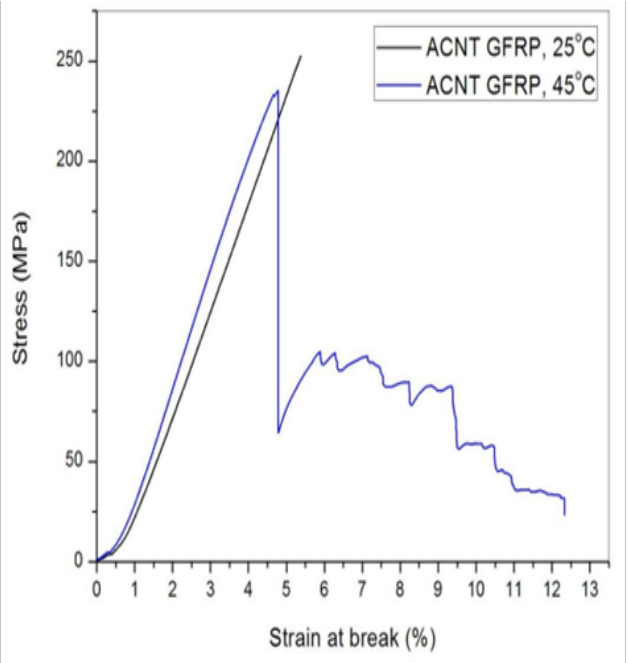

Figure 8 Effect of different infusion temperature on stress vs strain relationship for ACNT GFRP composites.

\section{Conclusion}

This study reports the effect of processing conditions on the dispersion and mechanical performance of GFRP composites reinforced with functionalized carbon nanotubes. On comparing the dispersion of nanotubes in the resin by either high-shear homogenizer or mechanical stirring, a drop was seen in the materials stiffness and strength i.e. $25 \%$ and $29 \%$ respectively, by using mechanical stirring technique. While no change, was observed for the tensile properties. Therefore, employing of high shear mixer technique resulted in breaking the nanotube agglomerates and further improved the interfacial bonding with the epoxy resin that enabled an effective stress transfer between the epoxy matrix and the amino-functionalized CNTs. 
Furthermore, experimental investigation illustrates that the addition of amino-functionalized CNTs in the low viscosity hardener shows better performance as compared to their dispersion in the resin. Also, lower amount of CNT agglomerates were observed in this case and the mechanical properties improved by approximately $30 \%$ in tensile modulus and approximately $10 \%$ in flexural strength and ILSS properties vis-à-vis ACNT dispersion in resin.

In addition, the infusion temperature of the resin mixture in VARIM has also shown profound effect on the mechanical behavior. In pure epoxy glass fiber composites, higher temperature has scored over room temperature specimens for all the properties studied. Whereas, in amino-functionalized nanotubes reinforced glass fiber composites, the tensile and flexural strength as well as Inter laminar shear strength (ILSS) properties increased for higher temperatures as compared to room temperature infusion. The slight improvement in the tensile strength is likely due to the presence of nanotubes in the matrix as they tend to bear the stress during its loading conditions. Additionally, the improvement in ILSS and flexural strength properties is highly due to the strong crosslinking network among amino-CNTs, resin and the glass fiber mat. Also, higher temperature of the resin mixture reduces its viscosity which further results in better dispersion of CNTs in the epoxy matrix, good wettability of the mixture with glass fiber mat, reduced porosity and agglomerates which act as stress-concentration sites thus, resulting in stronger interfacial adhesion.

\section{Acknowledgements}

The authors would like to thank Dr. Manoranjan Patri, Dr. Sangram K. Rath and Dr. Debdatta Ratna, NMRL for their guidance with the preparation of composites. The authors would like to thank SAIF Labs, Punjab University, Chandigarh, India for carrying out TEM analysis of the samples. Also, the help of SAI Labs, Thapar University, Patiala for the SEM images of fractured composite surfaces is duly acknowledged.

\section{Conflict of interest}

The author declares no conflict of interest.

\section{References}

1. Paszkiewicz S, Pilawka R, Zenker M, et al. Detailed study on interfacial interactions in epoxy composites cured with 1-buthylimidazole containing functionalized carbon nanotubes. Composite Interfaces. 2015;22(7):629-649.

2. Jarali CS, Patil SF, Pilli SC. Hygro-Thermo-Electric Properties of Carbon Nanotube Epoxy Nanocomposites with Agglomeration Effects. Mechanics of Advanced Materials and Structures. 2015;22(6):428-439.

3. Garg M, Sharma S, Mehta R. Pristine and amino functionalized nanotubes reinforced glass fiber epoxy composites. Composites Part A. 2015;76:92-101.

4. Rahman MM, Zainuddin S, Hosur MV, et al. Improvements in mechanical and thermo-mechanical properties of e-glass/epoxy composites using amino functionalized MWCNTs. Composite Structures. 2012;94:2397-2406.

5. Chandrasekaran VCS, Santare MH, Krishnan P, et al. Amino functionalization of MWCNTs and their effect on ILSS of hybrid nanocomposites. Composite Interfaces. 2011;18(4):339-355.

6. Sharma B, Mahajan S, Chhibber R, et al. Glass Fiber Reinforced Polymer-Clay Nanocomposites:Processing, Structure and Hygrothermal Effects on Mechanical Properties. Procedia Chemistr. 2012;4:39-46.
7. Sharma B, Mehta R, Chhibber R. Layered Silicates in fiber reinforced composites. Research Bulletin of Australian institute of High Energetic Materials. 2011;1:106-118.

8. Hosur MV, Rahman T, Brundidge-Young S, et al. Mechanical and Thermal Properties of Amine Functionalized Multi-walled Carbon Nanotubes Epoxy-Based Nanocomposite. Composite Interfaces. 2010;17(23):197-215

9. Li J, Fang Z, Tong L, et al. Improving dispersion of multiwalled carbon nanotubes in polyamide six composites through amino-functionalization. Journal of Applied Polymer Science. 2007;106:2898-2906.

10. Shen J, Huang W, Wu L, et al. The reinforcement role of different amino-functionalized multi-walled carbon nanotubes in epoxy nanocomposites. Composites Science and Technology. 2007;67:3041-3050.

11. Wang S, Liang Z, Liu T, et al. Effective amino functionalization of carbon nanotubes for epoxy polymer composites. Nanotechnology. 2006;17(6):1551-1557.

12. Ramanathan T, Fisher FT, Ruoff RS, et al. Amino-functionalized carbon nanotubes for binding to polymers and biological systems. Chem Matter. 2005;17(6):1290-1295.

13. Gojny FH, Schulte K. Functionalisation effect on the thermo-mechanical behavior of multi-wall carbon nanotube/epoxy composites. Compos Sci Technol. 2004;64:2303-2308.

14. Gao F. Clay/polymer composites: The story. Materials Today. 2004;7(11):50-55.

15. Eitan A, Kuiyang J, Dukes D, et al. Surface modification of multiwalled carbon nanotubes:toward the tailoring of the interface in polymer composites. Chemistry of Materials. 2003;15:3198-3201.

16. Alexandre M, Dubois P. Polymer-layered Silicate Nanocomposites:Preparation, properties and uses of a new class of materials. Mater Sci Eng Rep. 2000;28:1-63.

17. Gude MR, Prolongo SG, Urena A. Effect of the epoxy/amine stoichiometry on the properties of carbon nanotube.epoxy composites. J Therm Anal Calorim. 2012;108:717-723.

18. Rico M, Lopez J, Bouza R. Thermal behavior of blends based on a thermoplastic-modified epoxy resin with a crosslinking density variation. $J$ Therm Anal Calorim. 2011;105:599-606.

19. Lopez J, Rico M, Montero B, et al. Polymer blends based on an epoxy-amine thermoset and a themoplastic. $J$ Therm Anal Calorim. 2009;95:369-376

20. Bae J, Jang J, Yoon SH. Cure behavior of the liquid-crystalline epoxy/ carbon nanotube system and the effect of surface treatment of carbon fillers on cure reaction. Macromol Chem Phys. 2002;203:2196-2204.

21. Ma PC, Siddiquia NA, Maromb G, et al. Dispersion and Functionalization of Carbon Nanotubes for Polymer-Based Nanocomposites: A Review. Composites Part A-Appl Science and Manufturing. 2010;41(10):1345-1367.

22. Sham ML, Li J, Ma PC, et al. Cleaning and functionalization of polymer surfaces and nanoscale carbon fillers by UV/ozone treatment:A review. J Compos Mater. 2009;43:1537-1564

23. Ma PC, Kim JK, Tang BZ. Effects of silane functionalization on the properties of carbon nanotube/epoxy nanocomposites. Composites Science and Technology. 2007;67:2965-2972.

24. Sham ML, Kim JK. Surface functionalities of multi-walled carbon nanotubes after UV/ozone treatment and TETA treatments. Carbon. 2006;44:768-777

25. Ma PC, Kim JK, Tang BZ. Functionalization of carbon nanotubes using a silane coupling agent. Carbon. 2006;44:3232-3238. 
26. Shen $\mathrm{K}, \mathrm{Xu} \mathrm{H}$, Jiang $\mathrm{Y}$, et al. The role of carbon nanotube structure in purification and hydrogen adsorption. Carbon. 2004;42:2315-2322.

27. Mawhinney DB, Naumenko V, Kuznetsova A, et al. Infrared spectral evidence for the etching of carbon nanotubes: ozone oxidation at 298 K. J Am Chem Soc. 2000;122:2383-2384.
28. May CA. Epoxy resins:chemistry and technology. 2nd ed. USA: Marcel Dekker Inc; 1988: 907-909.

29. Kim M, Park YB, Okoli OI, et al. Processing, characterization, and modeling of carbon nanotube-reinforced multiscale composites. Composites Science and Technology. 2009;69(3-4):335-342. 